\title{
Deformation Mechanics of a Non-Linear Hyper-Viscoelastic Porous Material, Part II: Porous Material Micro-Scale Model
}

\author{
Christopher Salisbury $^{1} \cdot$ Duane Cronin $^{1} \cdot$ Fue-Sang Lien ${ }^{1}$
}

Received: 20 May 2015/ Accepted: 11 July 2015/Published online: 22 July 2015

(C) Society for Experimental Mechanics, Inc 2015

\begin{abstract}
Foam materials are widely used for energy absorbing applications, and are often addressed in a modeling environment at a macroscopic or continuum level by measuring the mechanical properties, which may be size dependent, and implementing the properties in a continuum-level constitutive model. However, foams are known to exhibit a characteristically low wave speed and an understanding of the deformation mechanics of foams at the micro-scale and dependence on morphology are essential to understand the performance of foam material in impact scenarios. In this study, experimental testing and finite element modeling were used to investigate a viscoelastic polychloroprene closed-cell foam at the cell level, subject to large deformation and high deformation rates. A numerical model was created with solid hexahedral elements and a repeated tetrakaidecahedron cell structure using measured foam cell size and wall thickness, and mechanical properties measured from non-porous polychloroprene. The finite element model predictions were evaluated using experimental compression tests on the foam material at high deformation rates. The enclosed nitrogen in the closed cell foam was modeled using an Arbitrary Lagrange-Eulerian method so that this contribution could be included or removed, and demonstrated the significant effect of the enclosed gas on the mechanical response of the foam. The foam cell dimensions were varied to investigate morphological factors including cell size, cell aspect ratio and cell wall thickness.
\end{abstract}

Duane Cronin

dscronin@uwaterloo.ca

1 Department of Mechanical and Mechatronics Engineering, University of Waterloo, 200 University Ave. West, Waterloo, ON, Canada
Increasing wall thickness, decreasing cell size and decreasing the cell aspect ratio resulted in increased material stiffness, with wall thickness having the most significant effect. Investigation of the wave transmission speed demonstrated a low value compared to the constituent materials, which was explained by the path of the stress wave through the foam structure and wave reflections within the cells, attenuating the stress wave. The consequence of this low wave speed was non-uniform deformation of the foam sample demonstrating that the measured mechanical properties of porous foams depend on the sample thickness, an important consideration for foam material testing and characterization.

Keywords Hyper-viscoelastic foam - Porous material . Explicit numerical modeling - Dynamic deformation . Wave speed · Foam morphology

\section{Introduction}

Many natural and man-made materials exhibit a porous structure. Historically, deformation mechanics in porous media have been viewed from the continuum scale where the characteristics are not investigated at the individual pore level, such as in the field of poroelasticity and foams [1-5]. Although these types of models are generally thought to adequately describe the mechanical properties of porous materials at a macro-scale, they do not address the micro-scale level, which is important in material design for energy absorption, wave propagation considerations, and to understand the effect of different morphologies on material response [6]. Several researchers have identified unique properties in porous materials such as a reduced wave speed [7-9] and attenuation of stress waves [10-13] when 
analyzing the deformation mechanics of both metallic and polymeric foams and at the pore level, where the current study considered a closed-cell polymeric foam.

Lakes et al. modeled buckling of the struts in an open cell structure for a single elastomeric foam structure [14] while Wismans et. al used 2D sections to model a closed cell polymeric foam [15]. A limitation of these studies was that the mechanical properties of the constituent materials were not known and so material parameters were assumed. Zhu et al. [16] developed a model of an open cell material using tetrakaidecahedrons to estimate the elastic modulus and Poisson's ratio for the foam. The material was assumed to be linear elastic and the elastic constants were found analytically while the effect of contained gas within the cells was assumed to be negligible. A similar study by Zhu et al. [17] investigated the high strain compression of polyurethane foams. This study examined the effect of buckling of the cell edges and compared predicted deformations to experiments; however, a plateau in the compressive stress-strain behavior was not predicted by this model, which was seen in the experiments. This model was further extended by Zhu et al. [18] to capture creep behavior using viscoelastic theory. Mills [19] used finite element analysis to predict the viscoelastic effects in the compression of polyurethane open cell foam during impact. Two models were investigated: the lattice structure model developed by Gibson and Ashby [20] and a "Wet" Kelvin model. Mills used a viscoelastic material model to simulate the polyurethane foam and suggested that the majority of the energy absorption could be attributed to the viscosity of the polymer. In 2007 Mills [21] further investigated the response of open cell polyurethane foam to predict the elastic modulus of the material as it changed during compression (densification of the foam).

Mills et al. [6] modeled closed cell polyethylene and polystyrene foams at a microscopic scale. In this study, the cells were modeled with an outer structure composed of shell elements of approximately $30 \mu \mathrm{m}$ in size, while the air was modeled with a special fluid cavity which acted on the outer shell. The author noted that he was unable to model the edges of the foam structure using solid elements. Although this study provided some important insights, limitations included: wave propagation effects were not investigated; a simple bilinear stress-strain curve scaled by $20 \%$ to match data at a strain rate of $50 \mathrm{~s}^{-1}$ was used that did not account for viscoelastic effects; and only a quarter section of the cells was modelled without consideration of the domain size.

The existing models and analyses have several limitations: the majority of the models are confined to open-cell foams and do not consider the effect of contained gas, the previous studies do not directly address wave effects such as predicting the characteristically low wave speed in foams or the importance morphological factors on the mechanical response of foam. The aim of this study was to develop and validate a numerical model of a hyperelastic, closed-cell, porous foam at the pore level that could then be used to investigate morphological factors and stress wave propagation effects.

\section{Methodology}

The creation of a pore-level foam model requires the constitutive properties of the material comprising the cell walls. It is challenging to accurately measure the properties of the cell walls at the pore scale due to their small size, and so a porous material whose cellular material properties could be measured using larger scale samples was desired. Polychloroprene foam (G207, Rubatex International LLC) was identified as an ideal candidate for this study. Initial tests of the polychloroprene foam indicated that it was elastomeric and was comprised of closed cells relatively equal in size. Additionally, sheets of solid or un-foamed polychloroprene rubber were available from the same material batch as the foamed material allowing the constituent properties of the un-foamed material to be measured, undertaken in Part I of this study. The density of the non-porous material was $1213.25 \mathrm{~kg} / \mathrm{m}^{3}\left(8.43 \mathrm{~kg} / \mathrm{m}^{3}\right.$ standard deviation) while the density of the foamed material was $335.05 \mathrm{~kg} / \mathrm{m}^{3}\left(3.46 \mathrm{~kg} / \mathrm{m}^{3}\right.$ standard deviation). Since the foaming process was done through a nitrogen injection process, not chemically as with many polymeric types of foam, it was assumed that the constituent material properties were not altered with the foaming process. Nonporous polychloroprene rubber was characterized using a hyper-viscoelastic constitutive model and implemented into an explicit finite element code (LS-Dyna, LSTC, Livermore CA) [22], described in Part I of this study [23, 24]. In brief, a computationally efficient additive hyperviscoelastic constitutive model was developed, implemented in an explicit finite element code, and demonstrated to model non-porous polychloroprene rubber across a wide range of deformation rates. In the present study, the physical structure of the foam (porous polychloroprene) was characterized through microscopic investigations and used to develop the numerical model geometry. Compression experiments performed on the foam were similar to those performed on solid material; however, it was not the intent of this study to characterize the foam at a macro scale level but instead to identify if the foamed material had a dependence on strain rate and to provide a set of experimental data against which the micro-scale numerical models could be assessed. The methodology to measure the compressive mechanic properties was the same as that used in Part I [23] of this study. The experimental test specimens 
were $10 \mathrm{~mm}$ in diameter and $4 \mathrm{~mm}$ in length and prepared using the same techniques as described in Part I of this study. The samples were tested at average strain rates ranging from 0.001 to $3000 \mathrm{~s}^{-1}$. At each loading rate a minimum of three tests were conducted and averaged to create a representative curve. Similar to the results in Part I of this study, the tests were repeatable with little variability, and the average curve was representative of the test data. A standard hydraulic test frame was used for compression testing at rates of $0.001,0.01,0.1$, and $1.0 \mathrm{~s}^{-1}$. Force was measured using a $2225 \mathrm{~N}$ (500 lbf) load cell (Strain Sert, model FL05U(C)-2SP) and displacement was measured with a Linear Variable Differential Transformer (Omega, type LD-320-7.5). A Polymeric Compressive Split Hopkinson Pressure Bar [23, 24] was used to test the foam at high deformation rates (2050 and $3000 \mathrm{~s}^{-1}$ ), with the benefit of having a relatively long rise time (approximately $100 \mu \mathrm{s}$ ), providing a gradual loading compared to metallic bars. This was important for testing the foamed material due to the characteristically low wave speed exhibited by foams in general. Using this apparatus, the sample was deformed at a constant velocity, and therefore a constant engineering strain rate. For the purposes of the current study, the bar end velocities were used as the numerical boundary conditions for the purpose of simulating the experiments.

The stress versus stretch response demonstrated increasing stiffness with increasing deformation rate for average curves of the foam material over the range of strain rates tested (Fig. 1). The shape of the curves indicated that this foam exhibited a response similar to the solid polycholoprene rubber. The hyperelastic nature of the foam prevented post-test inspection of the cellular structure to determine the uniformity of the cellular collapse; however, this was investigated using the numerical model.

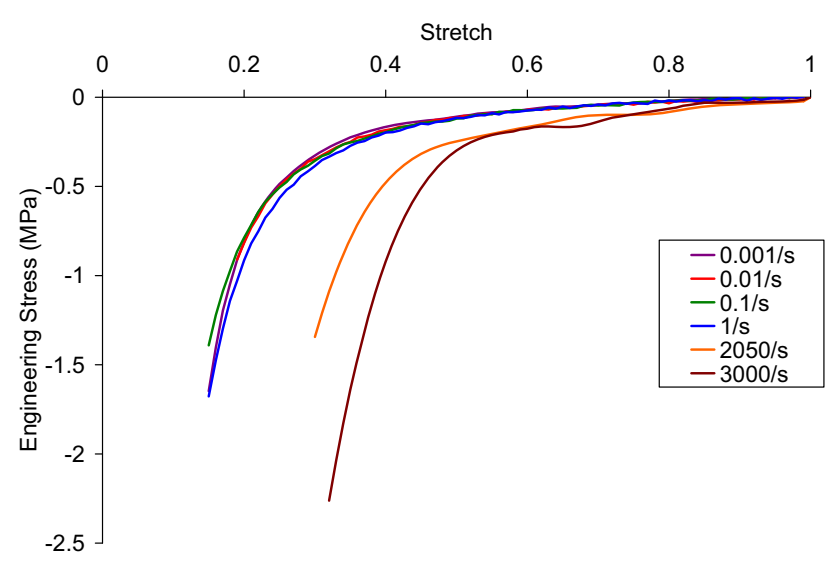

Fig. 1 Average stress-stretch curves for the polychloroprene foam over the strain rates tested

\section{Numerical Model Development}

\section{Foam Morphology}

In order to facilitate the microscopic analysis, a foam specimen with perpendicular faces was created. This was accomplished by first bonding the foam to an aluminum block. The foam was then cut using a rotary blade in a lathe with the aluminum block attached to the compound rest so that a constant feed rate could be achieved. The aluminum block was then rotated $90^{\circ}$ so that three perpendicular, flat faces were created approximately $7 \mathrm{~mm}$ in length. Distilled water was used as a lubricant to reduce friction and prevent tearing of the material during the cutting process.

Microscopic images were taken oriented with the X, Y and $\mathrm{Z}$ planes of the foam material. It was noted that the polychloroprene foam had a relatively consistent structure with similar-sized cells. A minimum of 100 measurements were taken in each plane to determine the characteristic length, $L_{c}$, and wall thickness of the cells (Fig. 2, Table 1). The geometric anisotropy of the cells, determined by taking the ratio of the inside dimensions of the cells along the principle axes, resulted in the aspect ratios of 1.06 in the $\mathrm{XY}$ plane, 1.79 in the $\mathrm{XZ}$ plane, and 1.56 in the $\mathrm{YZ}$ plane. Relatively consistent wall thickness was seen in the $\mathrm{XZ}$ and $\mathrm{YZ}$ planes with a slight increase in thickness in the $\mathrm{XY}$ plane. The $\mathrm{Z}$ direction was the foaming direction through the thickness of the sheet material and the cells exhibited a different aspect ratio than that seen in the cross-sectional $\mathrm{XY}$ plane. The overall average of all wall thickness measurements was $11.78 \mu \mathrm{m}$ with a standard deviation of $3.46 \mu \mathrm{m}$. The cells had a characteristic length of $154.77 \mu \mathrm{m}$ with a standard deviation of $52.79 \mu \mathrm{m}$.

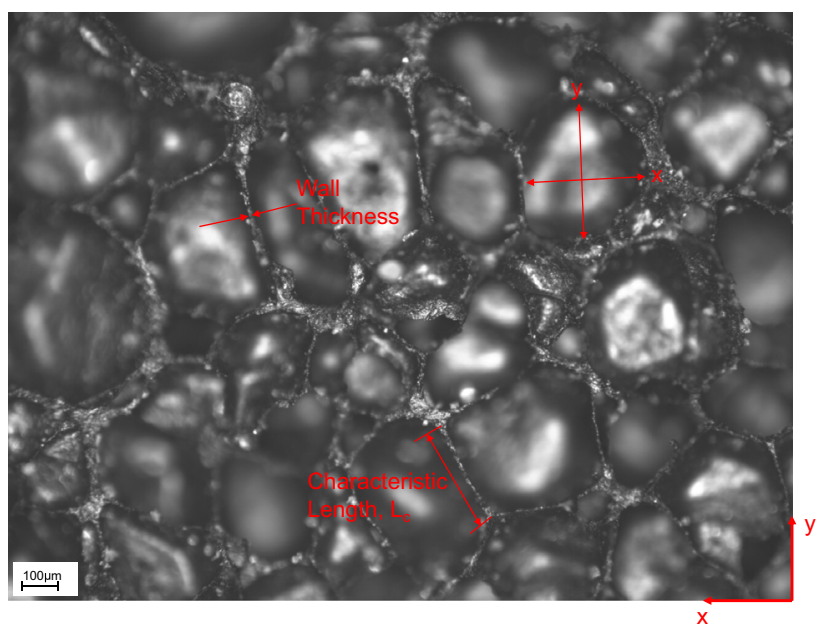

Fig. 2 Close-up view of foam micro-structure showing typical measurements 
Table 1 Measured values of wall thickness, characteristic length and aspect ratio for each plane for polychloroprene foam

\begin{tabular}{lllllll}
\hline Plane & Wall thickness $(\mu \mathrm{m})$ & Standard deviation & Characteristic length $(\mu \mathrm{m})$ & Standard deviation & Aspect ratio & Standard deviation \\
\hline $\mathrm{XY}$ & 13.4 & 4.8 & 180.4 & 56.5 & 1.06 & 0.20 \\
$\mathrm{XZ}$ & 11.6 & 3.0 & 135.9 & 49.7 & 1.79 & 0.41 \\
$\mathrm{YZ}$ & 11.2 & 2.8 & 148.9 & 42.0 & 1.56 & 0.28 \\
\hline
\end{tabular}

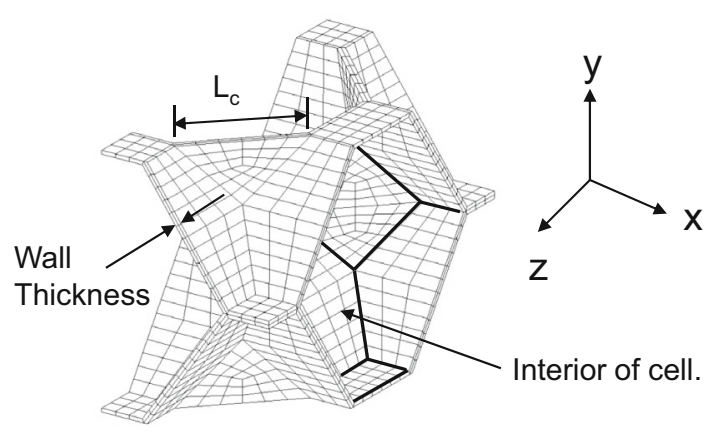

Fig. 3 Cross-sectional view of the base mesh

\section{Finite Element Mesh and Boundary Considerations}

The tetrakaidecahedron was identified as a candidate shape to model the pore structure of the foam following the work of Zhu et al. [16]. Microscopic analysis demonstrated that the cells were relatively equal in size and so, similar to the assumptions in [25], the pores of the foam were assumed to be space filling and equal in size. Figure 3 shows a crosssectional mesh of the base tessellation unit where $\mathrm{N}_{1}=3$ (number of elements along the half characteristic length) and $\mathrm{N}_{\mathrm{tt}}=1$ (number of elements through the half thickness of the wall). As evident in the figure, the cell walls along the diagonal included at least two cells through the thickness. The smallest element length of $6.1 \mu \mathrm{m}$ and total wall thickness of approximately $12.2 \mu \mathrm{m}$ corresponded to the foam measurements. Similarly, the characteristic length, $\mathrm{L}_{\mathrm{c}}$ (Fig. 3), was chosen to match that of the measurements taken. The base unit (Fig. 3) was then repeated through successive translations to achieve a tessellation with 2, 3, 2 repeats in the $\mathrm{x}, \mathrm{y}, \mathrm{z}$ directions (Fig. 4), respectively with a domain size of $1.28 \mathrm{~mm}$ by $1.35 \mathrm{~mm}$ by $1.28 \mathrm{~mm}(\mathrm{x}, \mathrm{y}, \mathrm{z})$. One of the inherent difficulties in modeling cellular materials numerically is the application of boundary conditions to the cut surface. To address this challenge, a surface on the top and bottom boundaries was created (Fig. 4). The surfaces were comprised of a layer, which was $2 \times \mathrm{N}_{\mathrm{tt}}$ elements thick, for a total thickness of $12 \mu \mathrm{m}$ and the square faces on the boundary which were only one element thick became three elements thick. The consequence of applying surfaces to the top and bottom of the

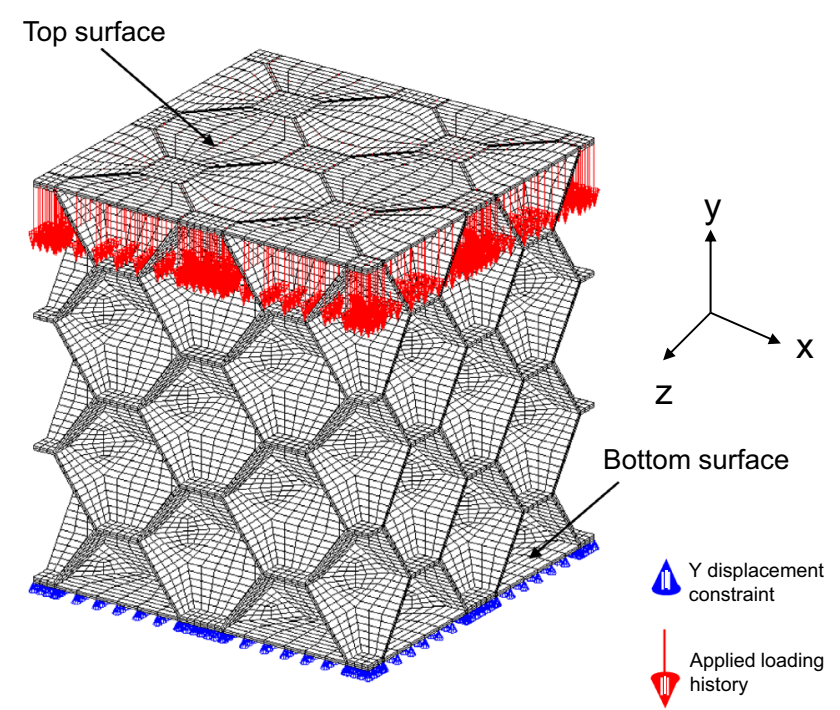

Fig. 4 A 2 by 3 by 2 tessellation of the cellular base unit showing top and bottom surfaces and boundary constraints

tessellation was that cells near the boundary were half the size of interior elements. The resulting model comprised approximately 48,000 solid elements. Nodes on the bottom surface of the model were constrained from moving in the $y$ direction, as indicated by the blue cones, and nodes on the top surface had an applied loading history (either displacement or velocity) in the y direction, as indicated by the red arrows (Fig. 4). The reaction forces at constrained nodes (blue) were monitored during the simulation for comparison.

The Arbitrary Lagrangian-Eulerian (ALE) algorithm (LS-Dyna, LSTC, Livermore CA) [22] was used to model the gas in the closed cells. The ALE method allowed the fluid domain to contract and expand arbitrarily independent of the fluid flow and was used to reduce the number of fluid elements required in the model to make the model computationally feasible. The movement of the fluid domain was controlled to ensure that the cells inside the tessellation were encompassed by the fluid mesh during the simulation. The Eulerian mesh was given a constant contraction in the $\mathrm{Y}$ direction and expansion in the $\mathrm{X}$ direction to allow the Lagrangian cells, which were moving through the Eulerian cells, to be fully encompassed at all times. The penalty 
coupling nature of the fluid structure interaction algorithm kept the fluid mass in each cell constant and thus the nitrogen was retained within the cells during the simulation. The fluid domain elements were initialized with a $101 \mathrm{kPa}$ pressure. A $101 \mathrm{kPa}$ pressure was applied to the surrounding surfaces so that the model was initially in equilibrium. The fluid structure interaction between the air and the constituent foam material was achieved through a penalty coupling algorithm as discussed in references [26] and [27].

In the present study, only the high deformation rate tests were modeled due to the simulation time required. Timescaling was investigated but was not applicable to this problem since stress wave effects were significant in the predicted material response. Several initial models were investigated to identify computational efficiencies of the analysis in comparison with the number of processors used. It was determined that by utilizing four parallel processors a reduction of computational time of approximately 2.5 times was achieved when compared to the case run with a single processor, while further increases in the number of processors did not provide significant benefit, highlighting the need for the computationally efficient constitutive model developed in Part I [23] of this study.

\section{Comparison of Numerical Models to Experimental Results}

The experimental results for the high deformation rate compression of the foamed polychloroprene rubber were used as a method of validation of the modeling techniques described in the previous section. For the simulations of the experiments, the domain size of the tessellation was 4 by 10 by 4 repeats of the base unit in the $\mathrm{x}, \mathrm{y}$ and $\mathrm{z}$ directions. This resulted in a cellular mesh that was approximately $2.6 \mathrm{~mm}$ by $4.5 \mathrm{~mm}$ by $2.6 \mathrm{~mm}$ in size which, when used with a mesh density of $\mathrm{N}_{\mathrm{tt}}=1$ and $\mathrm{N}_{1}=3$, resulted in approximately 578,000 solid elements. Although the length of the model of the cellular structure was similar to that of the experimental specimen, the cross-sectional area was significantly different with the area of the simulation being $6.51 \mathrm{~mm}^{2}$ while the area of the experimental sample was $72.0 \mathrm{~mm}^{2}$. If an equivalent area was used, 5,800,000 elements would have been required which was computationally prohibitive for the methods used in this study. The surface at the bottom of the model was constrained from movement in the y direction and the forces were recorded at each node. The loading history from the experiments was then applied to the top surface.

The loading history used for the simulation of the $2050 \mathrm{~s}^{-1}$ tests is shown in Fig. 5a. As identified in the figure, due to the rise time experienced in the Hopkinson
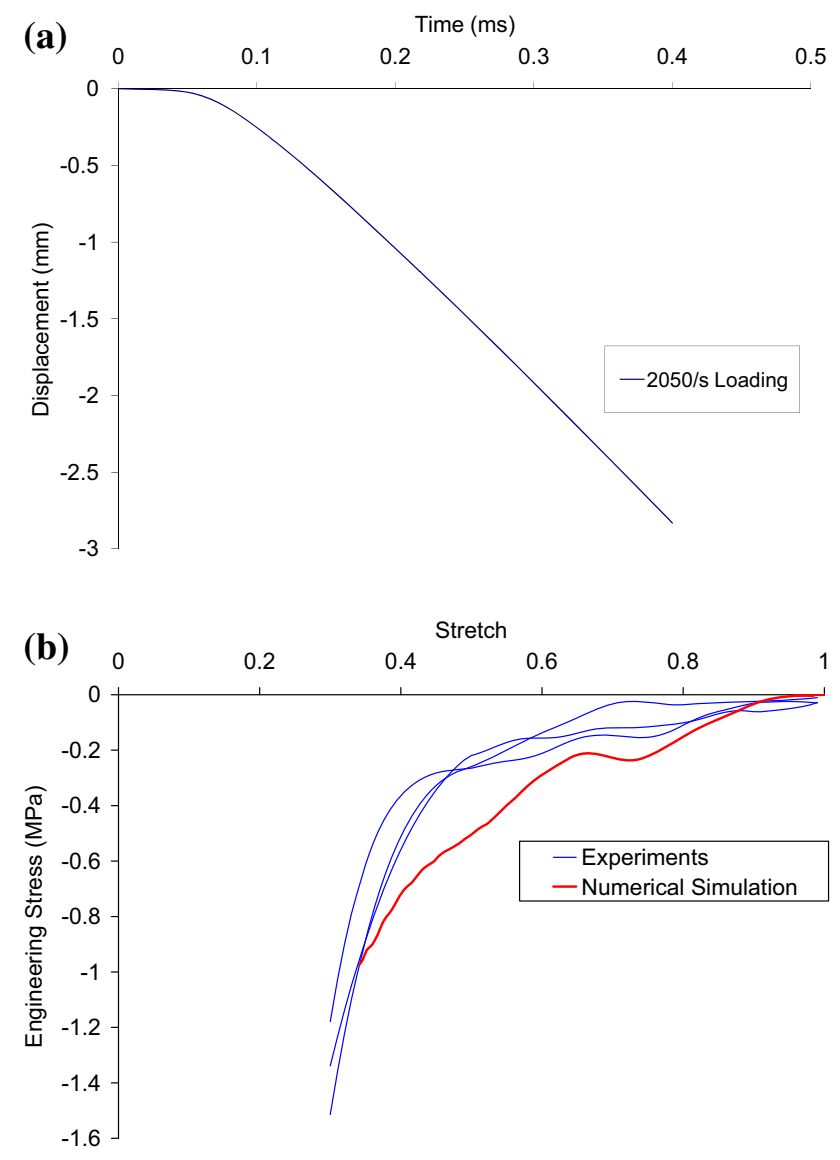

Fig. 5 a Loading history applied to the top surface of the model for the 2050/s case and b Comparison of numerical models and experiments for the 2050/s loading case

bar, the loading history had a non-linear portion where the velocity increased from zero to a relatively steady state (linear increase in displacement). Thus, the strain rates quoted were those in relation to the linear portion of the loading history from 0.1 to $0.375 \mathrm{~ms}$.

The results of the numerical model (Fig. 5b, red line) were compared to the experiments (blue lines) for the $2050 \mathrm{~s}^{-1}$ data, where the increase in the stress as the material was compressed was modeled as the stretch decreased from 1.0 to 0.65 (35\% compressive engineering strain). After this point, the cells began to collapse significantly with a corresponding increase in stress. The model over-predicted the stress through the 0.6-0.4 stretch regions and intersected the experimental results at a stretch of approximately 0.35 . This analysis $(4 \times$ $10 \times 4$ tessellation) required $780 \mathrm{~h}$ using 4 AMD 2352 processors. One numerical challenge was the significant reduction in time step corresponding to element deformation as the cells collapsed, making it computationally prohibitive to simulate large compressions, and so the simulation was terminated at the points indicated in the figures. 


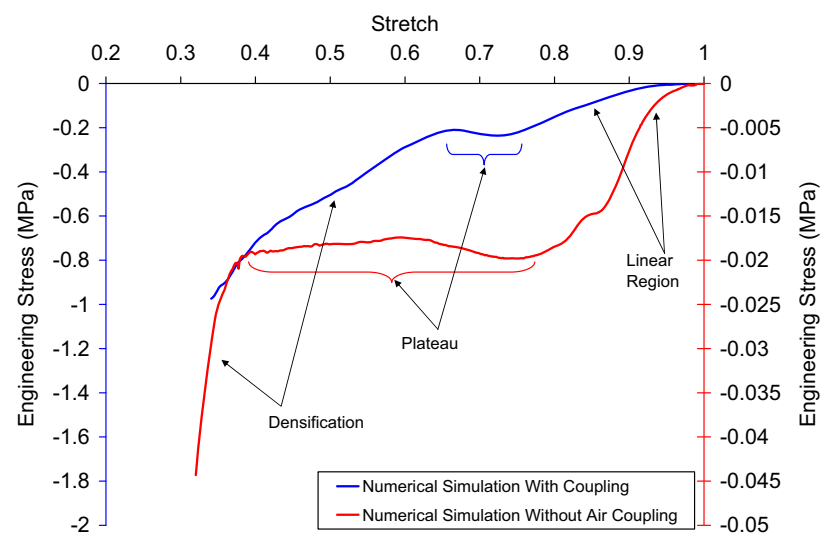

Fig. 6 Comparison of numerical model result for the coupled (left axis) and uncoupled (right axis) case at 2050/s

The effect of coupling the gas in the cells (blue line, Fig. 6, left ordinate axis) compared to the uncoupled simulation (red line, Fig. 6, right ordinate axis) was an order of magnitude increase in stress (note that the scales are different on the two axes). Additionally, the effect of the gas changed the shape of the curve significantly. Both curves exhibited a steep rise, plateau and densification region as identified by Gibson and Ashby [20]. However, the coupled simulation had a significantly reduced plateau region as referenced by the brace in the figure followed by gradual collapse of the pores owing to the presence of the gas in the cells. Additionally, the differences between the coupled and uncoupled simulations through the midplane of the model for the same displacement history demonstrated that the uncoupled simulation resulted in very little lateral expansion (i.e. a low Poisson's ratio, less than 0.01), whereas as the coupled simulation exhibited some lateral expansion (Poisson's ratio of 0.164).

\section{Parametric Analysis}

In order to identify relevant parameters which affect the foam mechanical response, several variants of the numerical model were created to investigate morphological factors including: wall thickness, cell size and geometric anisotropy. In order to reduce the computational burden, two reduced tessellations were created against which the various models were compared. The 2 by 3 by 2 mesh (Fig. 4) was chosen as the width (x), height (y) and depth (z) were nearly equal giving an aspect ratio $(\mathrm{x}=\mathrm{z}: \mathrm{y})$ of 0.94 for the overall domain. A different tessellation of 2 by 2 by 2 with an overall corresponding aspect ratio (x:y) of 1.4 was used to investigate the cell size effects. As will be discussed, this tessellation was chosen to ensure that the domain size remained the same while the number of cells inside the foam was varied.
To determine the appropriate mesh density of the model, three mesh densities (coarse $\mathrm{N}_{1}=1 \mathrm{~N}_{\mathrm{tt}}=1$, medium $\mathrm{N}_{1}=3 \mathrm{~N}_{\mathrm{tt}}=1$ and fine $\mathrm{N}_{1}=6 \mathrm{~N}_{\mathrm{tt}}=2$ ) were tested on a 2 by 3 by 2 tessellation with a $10 \mathrm{~mm} / \mathrm{ms}$ velocity input condition. Further refinement beyond this point was not considered as it was computationally prohibitive. This analysis indicated that the medium mesh $\left(\mathrm{N}_{1}=3 \mathrm{~N}_{\mathrm{tt}}=1\right)$ provided results comparable to the fine mesh with a significant reduction in computational time ( $135 \mathrm{~h}$ compared to $765 \mathrm{~h}$ ) and so the medium mesh was selected for the subsequent analyses.

\section{Wall Thickness Effects}

Five numerical models with increasing wall thickness by a factor of $2^{a}$, where $a$ was an integer ranging from 0 to 4 , were used to investigate the effect of wall thickness. The initial mesh, $2^{0}$, corresponded to the actual measured wall thickness while the $2^{4}=16$ case represented the largest mesh possible while still retaining pores in the material. This progression resulted in wall thicknesses of approximately 12, 24, 48, 96 and $192 \mu \mathrm{m}$ (Fig. 7a). For the 12, 24 and $48 \mu \mathrm{m}$ cases the mesh density was that used in the first analysis $\left(\mathrm{N}_{1}=3, \mathrm{~N}_{\mathrm{tt}}=1\right)$, for the $96 \mu \mathrm{m}$ case $\mathrm{N}_{\mathrm{tt}}$ was set to 2 , and for the $192 \mu \mathrm{m}$ case $\mathrm{N}_{\mathrm{tt}}$ was set to 4 . The mesh density was changed in this manner to prevent severely skewed elements from occurring. The relative densities for the wall thickness permutations are given in Table 2. As expected, increasing wall thickness resulted in increasing foam density.

The predicted force-stretch response for the different wall thicknesses (Fig. 7b) demonstrated reduced stretch to densification with increased wall thickness (and hence density). This is expected due to the reduced pore size resulting in an earlier onset of pore collapse. Additionally, upon inspection of the initial portion of the loading curve, the stretch, and hence time, before the stress wave reached the fixed end was reduced for increased wall thickness (96 and $192 \mu \mathrm{m}$ cases) .

\section{Cell Size Effects}

The model cell size was varied such that the overall domain size was maintained through modification of the cell size in multiples of 2 of the standard cell size. Therefore, a new base tessellation of 2 by 2 by 2 was created. If the cell size was halved, then the tessellation required to maintain the domain size was then 4 by 4 by 4 which corresponded to an increase in density by $72.5 \%$. Similarly, if the cell size was doubled, the tessellation required was 1 by 1 by 1 which corresponded to a decrease in density by $38 \%$. Three tessellations are shown (Fig. 8a) at a time of $0.011 \mathrm{~ms}$ after the start of deformation. As the 
(a)

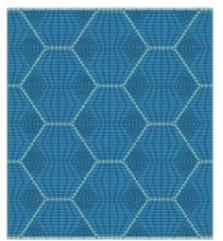

$12 \mu \mathrm{m}$ Wall

Thickness

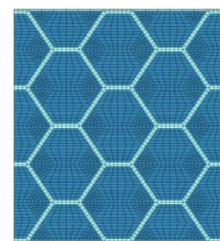

$24 \mu \mathrm{m}$ Wall Thickness

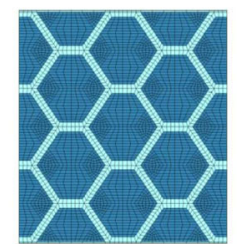

48um Wall Thickness

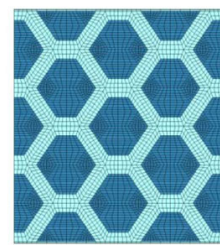

$96 \mu \mathrm{m}$ Wall Thickness

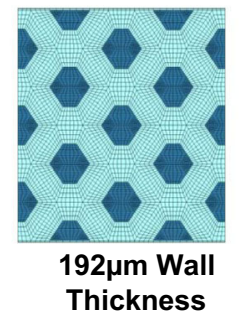

(b)

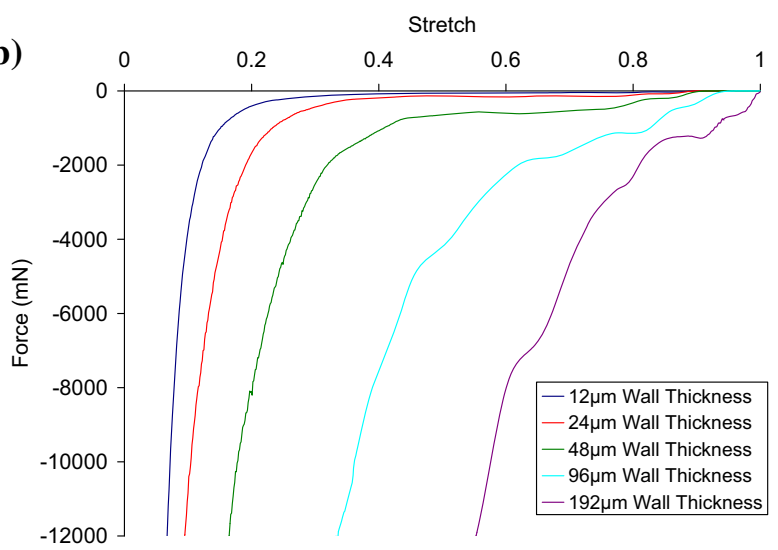

Fig. 7 a The five different models used to investigate the wall thickness effects. b The effect of wall thickness on the force response for five different models

Table 2 Wall thickness and porous foam relative density for the numerical model

\begin{tabular}{ll}
\hline Wall thickness $(\mu \mathrm{m})$ & Relative density [-] \\
\hline 12 & 1.0000 \\
24 & 1.7823 \\
48 & 3.2031 \\
96 & 5.5123 \\
192 & 8.3473 \\
\hline
\end{tabular}

cell size decreased (i.e. increase in density), the plateau level of force increased and the stretch at which the pores collapsed decreased. As the cell size decreased, a greater lateral expansion (higher Poisson's ratio) was observed. From these analyses it can be concluded that as the number of cells increases (resulting in an increase in density), so too does the stress level for the same amount of stretch. However, the particular material considered in this study was relatively insensitive for the measured cell size and (a)

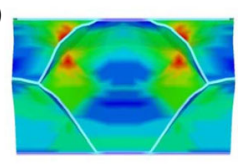

Double Cell Size 1 by 1 by 1

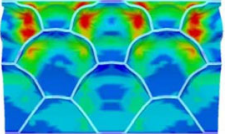

Standard Cell Size

2 by 2 by 2

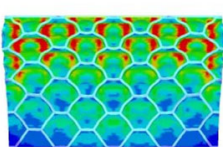

Half Cell Size

4 by 4 by 4

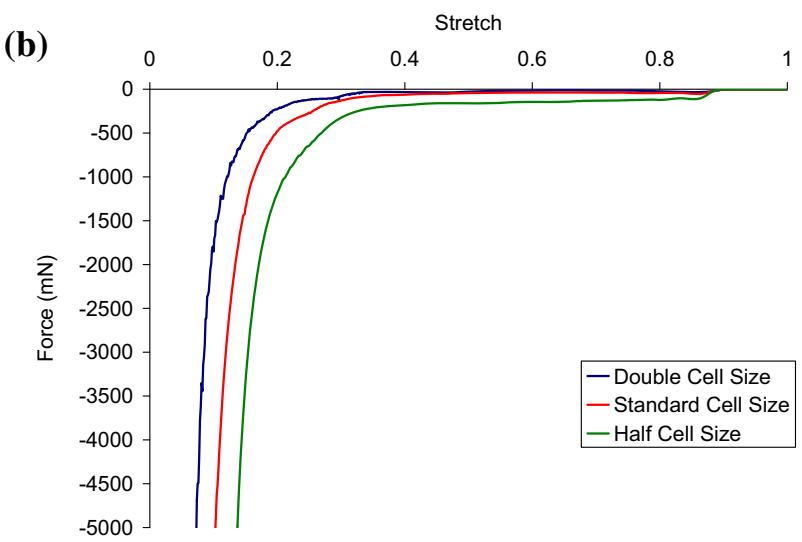

Fig. 8 a The three different models used to investigate cell size effects, contours of effective stress shown. b The effect of cell size on the force response for three different models loaded at $10 \mathrm{~m} / \mathrm{s}$

variability, with a large change in the cell size required to have a significant effect on the response of the material (Fig. 8b).

\section{Effect of Geometric Anisotropy}

Two different cell size aspect ratios were modeled to investigate the effect of anisotropy on the deformation behavior of the model. The aspect ratios (height: width $=$ depth, $\mathrm{y}: \mathrm{x}=\mathrm{z}$ ) chosen were 0.5 and 1.5 and compared to the standard 2 by 3 by 2 case. Note that the standard cell size had a small inherent aspect ratio with the height (distance from the square face to square face) being $0.438 \mathrm{~mm}$ and the width (as measure from the vertex of the intersecting hexagon) being $0.464 \mathrm{~mm}$ resulting in a height to width ratio of 1.08:1 (Fig. 9a). The models had the same tessellation and mesh density but due to the geometric anisotropic arrangement of the cells, the domain size was reduced from $1.38 \mathrm{~mm}$ for the standard case to approximately $0.7 \mathrm{~mm}$ for the 0.5 aspect ratio case and increased to $2.05 \mathrm{~mm}$ for the 1.5 aspect ratio case. This resulted in an increase in relative density of approximately $15 \%$ for the 0.5 aspect ratio case and $42 \%$ for the 1.5 aspect ratio case. Although the mass of the rubber for the 0.5 aspect ratio case was $58 \%$ of the standard case, the reduced volume resulted in an increase in density. The 1.5 aspect ratio and the standard case (Fig. 9b) exhibited nearly the same response while the 0.5 aspect ratio shows a reduced stretch prior to the cell collapse region compared to the standard case. As with the effect of cell size, significant 
(a)

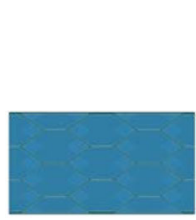

$0.5 \mathrm{H}: \mathrm{W}$

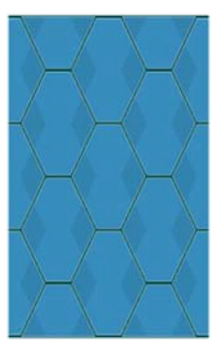

$1.5 \mathrm{H}: \mathrm{W}$ (b)

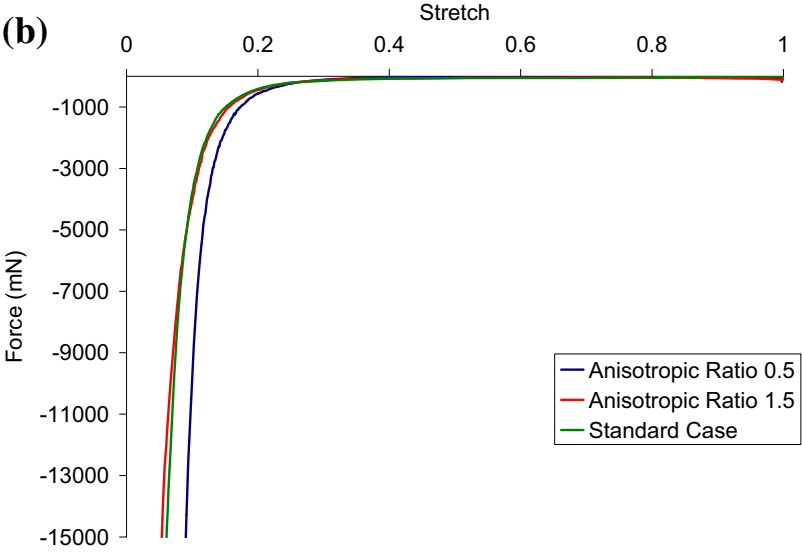

Fig. 9 a The three different models used to investigate effects of anisotropy. b The effect of anisotropy on the force response for three different models

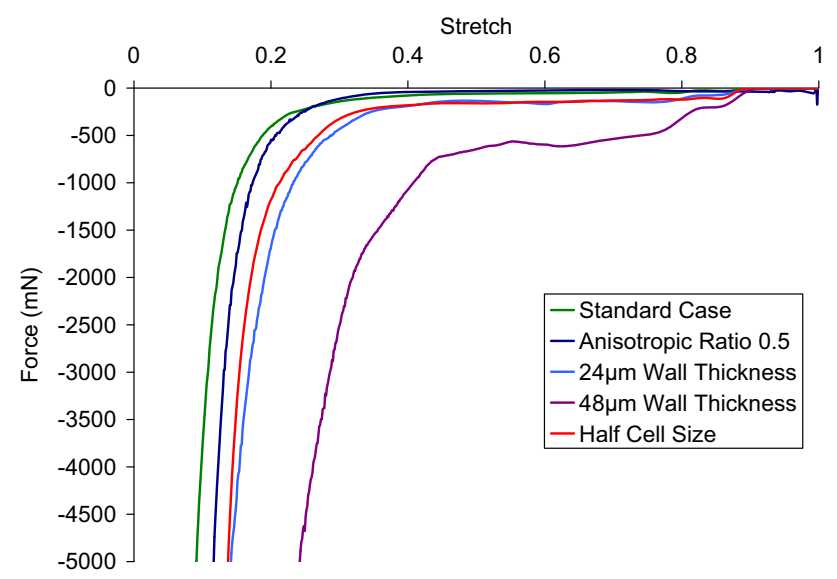

Fig. 10 The relative effects of the parameters compared in the models

changes in geometric anisotropy were required to have a measurable effect on the response of the material. The model identified that reducing the aspect ratio of the cells reduced the stretch to cell collapse.

A comparative summary of the effects is plotted in Fig. 10. which shows that increasing the wall thickness results in the largest change in material behavior for the parameters investigated.

\section{Stress Wave Propagation}

Porous materials exhibit low stress wave propagation velocities, often on the order of tens of meters per second compared to over one thousand meters per second for their constituent materials [28]. In the present case, the approximate sonic velocity of nitrogen at room temperature was $336 \mathrm{~m} / \mathrm{s}$ whereas in non-porous polychloroprene, the sonic velocity was approximately $1400 \mathrm{~m} / \mathrm{s}$ as discussed in references $[29,30]$. To illustrate the reduced wave speed in porous material in comparison to the constituent materials, a simulation of solid polychloroprene rubber and one of air were conducted with domains the same size as the 2 by 3 by 2 tessellation. The non-porous polychloroprene rubber had a $10 \mathrm{~m} / \mathrm{s}$ loading history applied to one surface. The simulation of the block of air was carried out by creating a block mesh of ALE elements and then having a plate of rubber elements with an applied velocity of $10 \mathrm{~m} / \mathrm{s}$ moving through it to provide a similar boundary condition. Figure 11a shows the initial propagation wave as it reaches the fixed end for the air compared to the porous structure of the foam at the same time. Figure $11 \mathrm{~b}$ shows the stress wave as it reaches the fixed end of the non-porous structure compared to the porous structure at the same time. As evident in the figures, the stress wave in the porous structure significantly lags that in the air and non-porous polychloroprene. From the simulations of the 2 by 3 by 2 tessellation, the apparent wave speed through the material ranged from $68.3 \mathrm{~m} / \mathrm{s}$ (using the peak of the effective stress measured in an element at the fixed end as the arrival time) to $115.5 \mathrm{~m} / \mathrm{s}$ (using the first detected change in effective stress).

Several factors were identified to contribute to the apparent low wave speed of the porous material. Considering the cellular material without any gas in the cells, applied loading resulted in the stress wave propagating through the cell walls, which varied in orientation to the applied loading. In comparison to the overall length of a pore, the stress wave had to propagate over a longer distance (e.g. around the pore) and the ratio of these lengths was a function of the cell size and isotropy. The initial ratio (length of stress wave path : overall length) of these lengths for the current analysis was 1.21:1 along the path through the square face and 1.26:1 along the mid-plane of the hexagonal faces which indicates that the wave had further to travel when propagating through the cell wall rather than through the cell. However, this analogy is simplistic in comparison to the actual propagation where the stress waves from multiple pores interact with each other creating multiple complex states including direct compression, shear and bending, all with different wave velocities. Additionally, the multiple interfaces between the air contained within the pore and the surrounding cellular structure caused multiple reflections and wave interactions. 
Fig. 11 Wave propagation through a cellular material compared to air $\mathbf{a}(\mathrm{t}=0.00337 \mathrm{~ms})$ and solid rubber $\mathbf{b}$ (contours of pressure shown $)(\mathrm{t}=0.00183 \mathrm{~ms})$

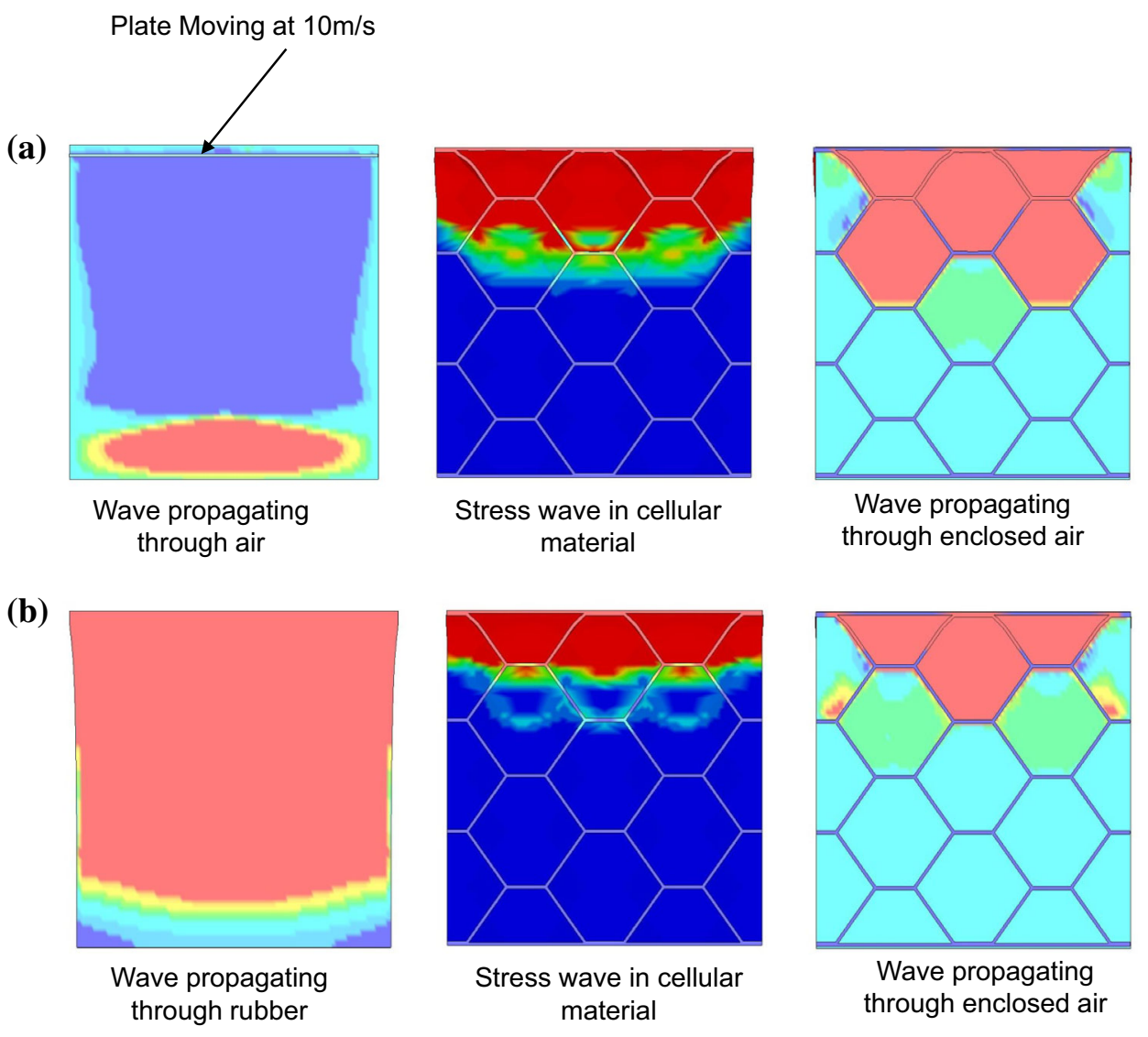

These mechanisms result in a material which quickly attenuates weak waves thereby reducing the overall wave propagation velocity that can be detected through disturbances in the stress or deformation of the elements.

The model demonstrated that there was a finite time required for the stress wave to propagate through a foam material, which is longer than the time required for wave transmission through the constituent materials (Fig. 11), and this effect can have a significant impact on the measured response of the material. In particular, when performing characterization experiments on porous materials the measured force will differ on the loaded versus the stationary sides of the foam due to the significant time required for transmission of the stress waves. This leads to non-uniform deformation of the foam (Fig. 8a) and a dependency on sample size so that traditional strain rate scaling based on length is not applicable to porous materials as it is currently applied to solid or non-porous materials. As shown in the model, there can be significant transient effects even at a loading rate of $10 \mathrm{~m} / \mathrm{s}$ that is approximately $15 \%$ of the initial stress wave velocity which is significant. This is an important consideration for the dynamic testing of foam materials. As an example, if the domain length of a piece of foam used in an application (e.g. foam layer in a helmet) is $25 \mathrm{~mm}$ thick and subjected to a loading rate of $28 \mathrm{~m} / \mathrm{s}$ (approximately $100 \mathrm{~km} / \mathrm{h}$ ) resulting in a nominal strain rate of $\sim 1100 / \mathrm{s}$, an experimental test on a material sample $9 \mathrm{~mm}$ in length at $10 \mathrm{~m} / \mathrm{s}$ ( $\sim 1100 / s)$ will provide a different response due to the smaller length and non-uniform deformation. However, as the loading rate decreases towards quasi-static rates, these transient effects are lessened.

\section{Summary}

A micro-scale finite element model of a closed cell hyperviscoelastic porous polychloroprene rubber was developed from measured foam morphology and high deformation/ high deformation rate mechanical properties of the constituent material measured in Part 1 of this study. The model was assessed using measured high deformation rate compressive properties of the foamed material. One limitation of the present study was the assumption that the mechanical properties of the material were not affected by the foaming process.

Through this model, the effect of the enclosed air, morphological factors and stress wave propagation factors were investigated. The model demonstrated that the effect of enclosed gas has a significant effect on the foam 
response, and must be modeled when investigating hyperelastic closed cell foams, a limitation of previous studies.

The primary limitation of this study was the small crosssectional area of foam modeled relative to the experimental test samples. The sample size investigated was a computational limitation and could have led to earlier cell consolidation due to lower constraint from adjacent cells; however, importantly the length of the model and test samples were approximately the same to ensure the transient wave effects and dependence on material thickness were accurately represented.

Several variations of the base model were considered to investigate morphological factors such as wall thickness, cell size, and anisotropy on the response of the foam. Of the morphological factors investigated, it was concluded that wall thickness had the most significant effect compared to cell size and cell dimension anisotropy. Future studies should include investigation of the effect of varying mechanical properties on foam response. Through investigating the mechanisms by which load is transmitted through the foam, stress path tortuosity and impedance coupling effects were shown to dissipate and attenuate stress waves propagating through this porous material, leading to a significant reduction in the apparent wave speed $(68.3 \mathrm{~m} / \mathrm{s})$ compared to the wave speed of the constituent materials $(1400 \mathrm{~m} / \mathrm{s}$ for polychloroprene, $336 \mathrm{~m} / \mathrm{s}$ for nitrogen). The model indicated that the sample deformed as the stress wave propagated through the cellular material illustrating the requirement to account for transient effects during experimental testing of porous materials. The non-uniform deformation exhibited by the material demonstrated that traditional strain rate scaling approaches are not applicable to porous materials at high deformation rates.

Acknowledgments The authors gratefully acknowledge the Natural Sciences and Engineering Research Council of Canada (NSERC) for financial support.

\section{References}

1. Reid SR, Peng C (1997) Dynamic uniaxial crushing of wood. J Impact Eng 19:531-570

2. Biot MA (1956) Theory of propagation of elastic waves in a fluid-saturated porous solid I. low-frequency range. J Acoust Soc Am 28(2):168-178

3. Biot MA (1956) Theory of propagation of elastic waves in a fluidsaturated porous solid I. higher frequency range. J Acoust Soc Am 28(2):179-191

4. Biot MA (1957) The elastic coefficients of the theory of consolidation. J Appl Mech 24:594-601

5. Biot MA (1962) Mechanics of deformation and acoustic propagation in porous media. J Appl Phys 33:1482-1498
6. Mills NJ, Stampfli R, Marone F, Bruhwiler PA (2009) Finite element micromechanics model of impact compression of closed-cell polymer foams. Int J Solids Struct 46(3-4):677-697

7. Kann KB (2005) Sound waves in foams. Colloids and Surf 263:315-319

8. Thuraisingham RA (1998) Sound speed in bubbly water at megahertz frequencies. Ultrasonics 36:767-773

9. Wilson PS (2005) Low-frequency dispersion in bubbly liquids. Acoust Res Lett 6(3):188-194

10. Kitagawa K, Takayama K, Yasuhara M (2006) Attenuation of shock waves propagating in polyurethane foams. Shock Waves 15:437-445

11. Berger PJ, Skuza EM, Ramsden CA, Wilkinson MHMH (2005) Velocity and attenuation of sound in the isolated fetal lung as it is expanded with air. J Appl Physiol 98(6):2235-2241

12. Umnova O, Attenborough K, Cummings A (2002) High amplitude pulse propagation and reflection from a rigid porous layer. Noise Control Eng. J. 50(6):204-210

13. Olim M, Van Dongen MEH, Kitamura T, Takayama K (1994) Numerical simulation of the propagation of shock waves in compressible open-cell porous material. Int J Multiphase Flow 20(3):557-568

14. Lakes R, Rosakis P, Ruina A (1993) Microbuckling instability in elastomeric cellular solids. J Mater Sci 28:4667-4672

15. Wismans JGF, Govaert LE, Van Dommelen JAW (2010) X-ray computed tomography-based modeling of polymeric foams: The effect of finite element model size on the large strain response. J Polym Sci, Part B: Polym Phys 48(13):1526-1534

16. Zhu XH, Knott JF, Mills NJ (1997) Analysis of the elastic properties of open-cell foams with tetrakaidecahedral cells. J Mech Phys Solids 45(3):319-343

17. Zhu XH, Mills NJ, Knott JF (1997) Analysis of the high strain compression of open-cell foams. J Mech Phys Solids 45(11-12): 1875-1904

18. Zhu XH, Mills NJ (1999) Modelling the creep of open-cell polymer foams. J Mech Phys Solids 47:1437-1457

19. Mills NJ (2006) Finite element models for the viscoelasticity of open-cell polyurethane foam. Cell Polym 25(5):293-316

20. Gibson LJ, Ashby MF (1997) Cellular Solids-Structure and Properties, 2nd edn. Cambridge University Press, Cambridge

21. Mills NJ (2007) The high strain mechanical response of the wet kelvin model for open-cell foams. Int J Solids Struct 44(1):51-65

22. Hallquist J (1998) LS-DYNA theoretical manual. LSTC, California

23. Salisbury CP, Cronin DS, Lien FS (2015) Deformation mechanics of a non-linear hyper-viscoelastic porous material, part I: testing and constitutive modeling of non-porous polychloroprene. J Dyn Behav Mater. Accepted July 2015

24. Salisbury CP (2011) On the Deformation mechanics of hyperelastic porous media. PhD Thesis.: University of Waterloo

25. Fung YC (1988) A model of the lung structure and its validation. J Appl Physiol 64(5):2132-2141

26. Olovsson L (2000) On the arbitrary Lagrangian-Eulerian finite element method. PhD Thesis.: Linköpings Universitet

27. Souli M, Ouashine A, Lewin L (2000) ALE formulation for fluidstructure interaction problems. Comput Methods Appl Mech Eng 190(5-7):659-675

28. Greer A (2006) Numerical modeling for the prediction of primary blast injury to the lung. masters Thesis, University of Waterloo

29. Millett JCF, Bourne NK (2001) Shock response of the elastomer, polycholorprene. J Appl Phys 85(1):2576-2579

30. Bourne NK, Millett JCF (2003) The high-rate response of an elastomer. Proc Roy Soc Lond A 459:567-576 